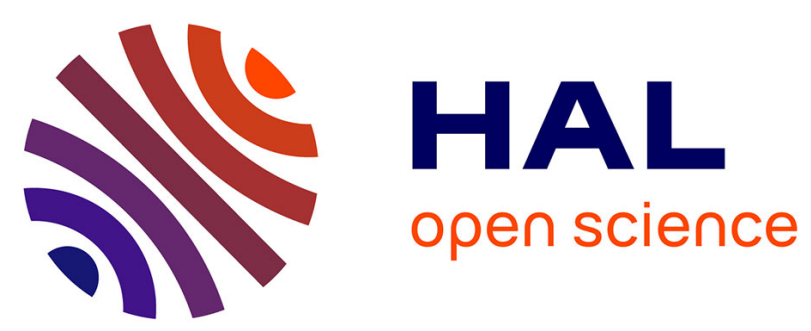

\title{
Model Predictive Control of Large-Dimension Cable-Driven Parallel Robots
}

\author{
João Cavalcanti Santos, Ahmed Chemori, Marc Gouttefarde
}

\section{To cite this version:}

João Cavalcanti Santos, Ahmed Chemori, Marc Gouttefarde. Model Predictive Control of LargeDimension Cable-Driven Parallel Robots. CableCon 2019: 4th International Conference on CableDriven Parallel Robots, Jun 2019, Krakow, Poland. pp.221-232, 10.1007/978-3-030-20751-9_19 . lirmm-02155794

\section{HAL Id: lirmm-02155794 https://hal-lirmm.ccsd.cnrs.fr/lirmm-02155794}

Submitted on 13 Jun 2019

HAL is a multi-disciplinary open access archive for the deposit and dissemination of scientific research documents, whether they are published or not. The documents may come from teaching and research institutions in France or abroad, or from public or private research centers.
L'archive ouverte pluridisciplinaire $\mathbf{H A L}$, est destinée au dépôt et à la diffusion de documents scientifiques de niveau recherche, publiés ou non, émanant des établissements d'enseignement et de recherche français ou étrangers, des laboratoires publics ou privés. 


\title{
Model Predictive Control of Large-Dimension Cable-Driven Parallel Robots
}

\author{
João Cavalcanti Santos, Ahmed Chemori, and Marc Gouttefarde \\ LIRMM, University of Montpellier, CNRS, Montpellier, France \\ \{joao.cavalcanti-santos, ahmed.chemori, marc.gouttefarde\}@lirmm.fr
}

\begin{abstract}
A Model Predictive Control (MPC) strategy is proposed in this paper for large-dimension cable-driven parallel robots working at low speeds. The latter characteristic reduces the non-linearity of the system within the MPC prediction horizon. Therefore, linear MPC is applied and compared with two commonly used strategies: Sliding mode control and PID+ control. The simulations aim at comparing disturbance rejection performances and the results indicate a superior performance of the proposed controller. Indeed, MPC takes into account control limits (cable tension limits) directly in the control design which allows the controller to better exploit the robot capabilities. In addition, actuation redundancy is resolved as an integral part of the control strategy, instead of calculating the desired wrench and then applying a tension distribution method.
\end{abstract}

Keywords: Cable-driven parallel robots, model predictive control, disturbance rejection

\section{Introduction}

A rather typical control strategy for Cable-Driven Parallel Robots (CDPRs) is the combination of a linear feedback control with computed torque applied as a feedforward term [1]. Indeed, the computed torque (also known as feedback exact linearization) enables the application of usual linear control methods $[2,3]$. Besides, Sliding Mode Control (SMC) is an advanced nonlinear feedback control that has been implemented successfully in CDPRs [4-7]. The main advantages of SMC are the possibility to attain finite time convergence, simple implementation and robustness to uncertainties. However, recent and advanced SMC methods still present chattering issues in experimental setups [6] even if some previous studies presented methods in order to reduce it [7].

Since a fully-constrained CDPR has more cables than degrees of freedom (DOF), there are infinitely many possible combinations of cable tensions generating a desired wrench. The choice of one of these combinations is an actuation redundancy resolution problem where cable tension lower and upper limits should be taken into account. The lower limit is a positive tension to avoid cable slackness. The upper limit is set in order to account for the mechanical 
limitations of the cables, motors, etc. Several previous works deal with CDPR actuation redundancy resolution, e.g. [8,9].

Usually, the control strategy and the resolution of actuation redundancy are addressed separately. This paper proposes the use of Model Predictive Control (MPC) as the control strategy, which has the advantage of solving the tension distribution problem as an integral part of the control strategy. MPC is a feedback control design which, at each decision instant, computes the sequence of future controls inputs that optimizes a cost function satisfying the system constraints. The cost function is formed by a weighted sum of individual costs (tracking errors, control input and other performance measures). MPC is considered as one of the most general way of posing a control problem in the time domain [10, 11]. It defines an optimized admissible control sequence if the considered model is sufficiently close to reality. Moreover, control limits can be directly handled, which is an important advantage since the optimized performance is often obtained with active constraints. If the MPC is applied to a linear optimization problem without constraints, the solution is analytic, e.g. in [12], where Generalized Predictive Control (GPC) is successfully applied to a medical. Nevertheless, constraints [13] and nonlinear systems [14] have been successfully addressed as well.

This paper presents the design, implementation and simulation of an MPC scheme for motion control of large-dimension CDPRs. To the best of our knowledge, MPC has never been used to control CDPRs. Several applications of CDPRs involve a large workspace and relatively low velocities of the mobile platform [15-18]. Hence, within a reasonable prediction horizon, the CDPR dynamics may be approximated as being a linear time invariant system. Based on this assumption, linear MPC is applied in this paper. Simulation results compare the performances obtained with the proposed MPC and two other control strategies, namely SMC and PID+ Controller [19]. The performance evaluation focuses mainly on external disturbance rejection.

The paper is organized as follows. The modeling of a spatial CDPR is introduced in Section 2. Two state-of-the-art control schemes, SMC and PID+ Control, and the proposed MPC strategy are introduced in Section 3. These three methods are compared through numerical simulations in Section 4. Conclusions and future works are drawn in Section 5.

\section{Dynamic Modeling of CDPRs}

The dynamic modeling of a spatial CDPR is presented in this section. The control schemes introduced later in Section 3 are based on this model. Figure 1 illustrates a CDPR with the notations used in its kinematic modeling. The robot consists of a $n$-DOF mobile platform driven by $m$ cables. The spatial case where $n=6$ is considered and the $m$ cables $(m \geq 6)$ are considered massless and inextensible. Each cable has one end attached to the platform and the other end wound on a winch drum. The cables are responsible for transmitting to the platform the efforts applied by the winches. Cable tensions $\tau$ applied on 
the platform generates the wrench $\mathbf{f}$. These variables are related linearly by the wrench matrix $\mathbf{W}$, so that $\mathbf{f}=\mathbf{W} \boldsymbol{\tau}[9]$.

The length $l_{i}$ is defined as the distance between the drawing point $A_{i}$ and and the attachment point $B_{i}$. Point $A_{i}$ is the drawing point defined by the pulley attached to the base frame. This point is considered as being fixed. Point $B_{i}$ is the attachment point of cable $i$ on the platform. The cable length vector is $\mathbf{l}=\left[l_{1}, \ldots, l_{m}\right]^{T}$.

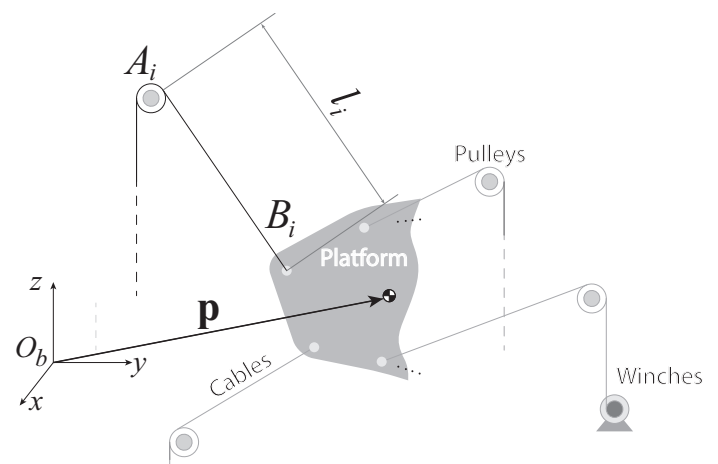

Fig. 1. Illustration of a CDPR with notations for kinematic modeling

Let $\mathbf{x}, \dot{\mathbf{x}}, \ddot{\mathbf{x}}$ be the platform pose, velocity and acceleration, respectively ${ }^{1}$. The pose (position and orientation) of the platform is then defined by $\mathbf{x}=\left[\mathbf{p}^{T}, \boldsymbol{\psi}^{T}\right]^{T}$. $\mathbf{p}$ is the position vector of the reference point of the platform. Vector $\boldsymbol{\psi}$ defines the orientation of the platform. Typically, it is composed of three Euler angles.

Using Newton-Euler formalism, the dynamics of the platform can be written as $[20]$

$$
\mathbf{M}(\mathbf{x}) \ddot{\mathbf{x}}+\mathbf{C}(\mathbf{x}, \dot{\mathbf{x}}) \dot{\mathbf{x}}=\mathbf{g}(\mathbf{x})+\mathbf{f}
$$

where matrices $\mathbf{M}$ and $\mathbf{C}$ are given by

$$
\mathbf{M}(\mathbf{x})=\left[\begin{array}{cc}
m_{p} \boldsymbol{I}_{3} & -m_{p} \hat{\mathbf{c}} \\
m_{p} \hat{\mathbf{c}} & \mathbf{H}
\end{array}\right], \quad \mathbf{C}(\mathbf{x}, \dot{\mathbf{x}}) \dot{\mathbf{x}}=\left[\begin{array}{c}
m_{p} \hat{\boldsymbol{\omega}} \hat{\boldsymbol{\omega}} \mathbf{c} \\
\hat{\boldsymbol{\omega}} \mathbf{H} \boldsymbol{\omega}
\end{array}\right]
$$

Scalar $m_{p}$ is the platform mass and $\boldsymbol{I}_{3}$ is the identity matrix of dimension 3 . The present model considers that the platform geometric center and its center of mass may not be coincident and, accordingly, $\mathbf{c}=\left[\begin{array}{lll}c_{x} & c_{y} & c_{z}\end{array}\right]^{T}$ is the vector going from the platform geometric center to its center of mass. Matrices $\hat{\boldsymbol{\omega}}$ and $\hat{\mathbf{c}}$ are the skew-symmetric matrices associated to $\boldsymbol{\omega}$ and $\mathbf{c}$, respectively, with $\boldsymbol{\omega}$ the angular velocity of the platform. The matrix $\mathbf{H}$ is defined as $\mathbf{H}=\mathbf{I}+$ $m_{p} \hat{\mathbf{c}} \hat{\mathbf{c}}^{T}$ where $\mathbf{I}$ is the platform inertia matrix relative to its center of mass.

\footnotetext{
${ }^{1}$ We highlight that $\dot{\mathbf{x}} \neq \frac{d \mathbf{x}}{d t}$, since angular velocity is not equal to the derivative of the vector of Euler angles. Similarly, $\ddot{\mathbf{x}} \neq \frac{d^{2} \mathbf{x}}{d t}$.
} 
The vector of gravitational forces is $\mathbf{g}(\mathbf{x})=m_{p} g\left[\begin{array}{llllll}0 & 0 & -1 & -c_{y} & c_{x} & 0\end{array}\right]^{T}$. The wrench applied by the cables on the platform is $\mathbf{f}=\mathbf{W} \boldsymbol{\tau}$.

\section{Proposed Control Schemes}

The goal of this paper is to compare MPC performance to those obtained with strategies commonly used for CDPRs. Namely, MPC is compared to: (i) A linear PID+ controller [19], based on the controller proposed in [20], and (ii) SMC. These two strategies were recently validated experimentally, demonstrating applicability and good performances. A brief description of these control methods is presented in the following section.

\subsection{Background on the State of the Art Controllers}

For a given reference trajectory in time $t_{i} \leqslant t \leqslant t_{f}$, the desired poses, velocities and accelerations are denoted as $\mathbf{x}_{d}(t), \dot{\mathbf{x}}_{d}(t)$ and $\ddot{\mathbf{x}}_{d}(t)$, respectively. At a given instant $t$, the error in the Cartesian space is expressed as $\mathbf{e}_{x}(t)=\mathbf{x}_{d}(t)-\mathbf{x}(t)$. Similarly, the error in joint space is $\mathbf{e}_{j}(t)=\mathbf{l}_{d}(t)-\mathbf{l}(t)$, where $\mathbf{l}_{d}(t)$ is the vector of desired cable lengths obtained with the inverse kinematics.

PID+: The PID+ control strategy applies the following wrench

$$
\mathbf{f}=\mathbf{M}(\mathbf{x}) \ddot{\mathbf{x}}_{d}+\mathbf{C}(\mathbf{x}, \dot{\mathbf{x}}) \dot{\mathbf{x}}_{d}-\mathbf{g}(\mathbf{x})+\mathbf{W}\left(\mathbf{K}_{p} \mathbf{e}_{j}+\mathbf{K}_{i} \int_{t_{i}}^{t} \mathbf{e}_{j}(\tau) d \tau+\mathbf{K}_{d} \dot{\mathbf{e}}_{j}\right)
$$

where $\mathbf{K}_{p}, \mathbf{K}_{i}$ and $\mathbf{K}_{d}$ are diagonal matrices containing the linear feedback PID gains.

Sliding Mode Control: The SMC strategy defines a sliding surface $\mathbf{s}=\mathbf{e}_{x}+\mathbf{C}_{e} \dot{\mathbf{e}}_{x}$, with $\mathbf{C}_{e}=\operatorname{diag}\left(c_{1}, \ldots, c_{n}\right)$. The wrench to be applied on the platform is

$$
\mathbf{f}=\mathbf{M}\left(\ddot{\mathbf{x}}_{d}+\mathbf{C}_{d}\left(\dot{\mathbf{x}}_{d}-\dot{\mathbf{x}}\right)+\mathbf{K} \operatorname{sat}(\mathbf{s})+\mathbf{Q} \mathbf{s}\right)+\mathbf{C}(\mathbf{x}, \dot{\mathbf{x}}) \dot{\mathbf{x}}_{d}-\mathbf{g}(\mathbf{x})
$$

where $\mathbf{K}, \mathbf{Q}$ and $\mathbf{C}_{d}$ are diagonal gain matrices. The function sat(s) is a continuous approximation of the sign function. Each component of this vector-valued function is calculated as follows

$$
\operatorname{sat}\left(s_{i}\right)=\left\{\begin{array}{ccc}
1, & \text { if } & s_{i}>\Delta \\
\frac{s_{i}}{\Delta}, & \text { if } & \left|s_{i}\right| \leqslant \Delta \\
-1, & \text { if } & s_{i}<\Delta
\end{array}\right.
$$

The resulting function presents the same output than the $\operatorname{sign}(s)$ function except for the interval $-\Delta \leqslant s \leqslant \Delta$ in which a linear interpolation eliminates the discontinuity. 
Redundancy Resolution: The two above control strategies define wrench $\mathbf{f}$. The final control output is the vector of cable tensions $\boldsymbol{\tau}$ (or motor torques). For fully-constrained CDPRs, $m>n$ and actuation redundancy shall be resolved to determine $\boldsymbol{\tau}$ being given $\mathbf{f}$, i.e., the equation system $\mathbf{W} \boldsymbol{\tau}=\mathbf{f}$ is underdetermined and an appropriate vector of cable tensions $\boldsymbol{\tau}$ shall be determined among the infinitely many possible ones (assuming a non-singular pose). In this work, the following common optimization problem is used to resolve actuation redundancy

$$
\begin{aligned}
\min _{\boldsymbol{\tau}}\|\boldsymbol{\tau}\|_{2} & \\
\text { s.t. } \mathbf{W} \boldsymbol{\tau} & =\mathbf{f} \\
\boldsymbol{\tau}_{\text {min }} & \leqslant \boldsymbol{\tau} \leqslant \boldsymbol{\tau}_{\text {max }}
\end{aligned}
$$

Using (6), the 2-norm of $\boldsymbol{\tau}$ is minimized. As constraints, the tension distribution shall generate the desired wrench (7) and the cable tensions shall be in an admissible interval (8). The constraint (8) is necessary mainly for two reasons: avoiding cable slackness $\left(0 \leqslant \boldsymbol{\tau}_{\min } \leqslant \boldsymbol{\tau}\right)$ and not violating mechanical limitations of the cables, motors, etc $\left(\boldsymbol{\tau} \leqslant \boldsymbol{\tau}_{\text {max }}\right)$.

In some cases, the wrench demanded by the controller is not feasible. In the space of cable tensions, the intersection of the subspaces defined by the constraints (7) and (8) is empty. Another strategy should then be defined and the following optimization problem is proposed

$$
\begin{aligned}
& \min _{\boldsymbol{\tau}}\|\mathbf{W} \boldsymbol{\tau}-\mathbf{f}\|_{\mathbf{P}} \\
& \text { s.t. } \tau_{\min } \leqslant \boldsymbol{\tau} \leqslant \tau_{\max }
\end{aligned}
$$

where the subscript $\mathbf{P}$ indicates that the 2-norm is calculated with a weighting positive definite diagonal matrix $\mathbf{P}$, which is necessary since $\mathbf{f}$ has components with inconsistent units (forces and moments).

\subsection{Proposed Model Predictive Controller (MPC)}

A model predictive control strategy is proposed in the present section. The continuous model (1) may be approximated as a discrete-time system

$$
\mathbf{y}(t+\Delta t)=\left[\begin{array}{c}
\mathbf{x}(t+\Delta t) \\
\dot{\mathbf{x}}(t+\Delta t)
\end{array}\right]=\mathbf{A}(t) \mathbf{y}(t)+\mathbf{B}(t) \boldsymbol{\tau}(t)+\mathbf{v}(t)
$$

where the vector $\mathbf{v}$ and the matrices $\mathbf{A}$ and $\mathbf{B}$ are given by

$$
\begin{aligned}
\mathbf{v} & =\left[\begin{array}{c}
\mathbf{0}_{n \times 1} \\
\Delta t \mathbf{M}^{-1} \mathbf{g}
\end{array}\right] \\
\mathbf{A} & =\left[\begin{array}{cc}
\mathbf{I}_{n} & \Delta t \mathbf{I}_{n} \\
\mathbf{0}_{n \times n} & \mathbf{I}_{n}-\Delta t \mathbf{M}^{-1} \mathbf{C}
\end{array}\right] \\
\mathbf{B} & =\left[\begin{array}{c}
\mathbf{0}_{n \times m} \\
\Delta t \mathbf{M}^{-1} \mathbf{W}
\end{array}\right],
\end{aligned}
$$


where time $t$ has been omitted, being given that $\mathbf{v}, \mathbf{A}$ and $\mathbf{B}$ are calculated at time $t$.

MPC predicts the states over a given horizon $t+N \Delta t$. Equation (10) is applied $N$ times in order to obtain $\mathbf{y}(t+i \Delta t)$, with $i=1, \ldots, N$. As mentioned earlier, the proposed MPC strategy is applied to large-dimension CDPRs moving at low velocities. Therefore, for a small horizon $N \Delta t$, the linear time varying system (10) may be approximated by a linear time invariant system. Matrix $\mathbf{A}$ is considered as $\mathbf{A}(t+i \Delta t)=\mathbf{A}(t), i=1, \ldots, N$. Similarly, $\mathbf{B}$ and $\mathbf{v}$ are considered constant over the MPC optimization horizon.

With this approximation, linear MPC can be applied. A vector $\mathbf{Y}(t)$ containing the states over the prediction horizon is calculated as follows

$$
\begin{aligned}
& \mathbf{Y}(t)=\left[\begin{array}{c}
\mathbf{y}(t+\Delta t) \\
\vdots \\
\mathbf{y}(t+N \Delta t)
\end{array}\right]=\underbrace{\left[\begin{array}{c}
\mathbf{A} \\
\mathbf{A}^{2} \\
\vdots \\
\mathbf{A}^{N}
\end{array}\right]}_{\mathbf{D}} \mathbf{y}(t)+\underbrace{\left[\begin{array}{ccccc}
\mathbf{B} & 0 & \cdots & 0 \\
\mathbf{A B} & \mathbf{B} & 0 & \ldots & 0 \\
\mathbf{A}^{2} \mathbf{B} & \mathbf{A B} & \mathbf{B} & 0 & \ldots \\
\vdots & & & & \\
\mathbf{A}^{N-1} \mathbf{B} & \mathbf{A}^{N-2} \mathbf{B} & \ldots & & \mathbf{B}
\end{array}\right]}_{\mathbf{E}} \underbrace{\left[\begin{array}{c}
\boldsymbol{\tau}(t) \\
\boldsymbol{\tau}(t+\Delta t) \\
\vdots \\
\boldsymbol{\tau}(t+(N-1) \Delta t)
\end{array}\right]}_{\mathbf{U}(t)}+\ldots \\
& \ldots+\underbrace{\left[\begin{array}{c}
\mathbf{v} \\
\mathbf{A} \mathbf{v}+\mathbf{v} \\
\vdots \\
\mathbf{A}^{N-1} \mathbf{v}+\mathbf{A}^{N-2} \mathbf{v}+\ldots+\mathbf{v}
\end{array}\right]}_{\mathbf{F}}
\end{aligned}
$$

Similarly, the reference trajectory $\mathbf{y}_{d}=\left[\mathbf{x}_{d}^{T}, \dot{\mathbf{x}}_{d}^{T}\right]^{T}$ should be defined over the prediction horizon

$$
\mathbf{w}(t)=\left[\begin{array}{c}
\mathbf{y}_{d}(t+\Delta t) \\
\mathbf{y}_{d}(t+2 \Delta t) \\
\vdots \\
\mathbf{y}_{d}(t+N \Delta t)
\end{array}\right]
$$

For a given current state $\mathbf{y}(t)$, the control is responsible for finding a trade-off between minimizing the predicted error $\mathbf{w}(t)-\mathbf{Y}(t)$ and minimizing the control effort $\mathbf{U}(t)$. To this end, the following cost function can be considered

$$
J(\mathbf{y}, \mathbf{U})=(\mathbf{w}-\mathbf{Y})^{T} \mathbf{K}_{Y}(\mathbf{w}-\mathbf{Y})+\mathbf{U}^{T} \mathbf{K}_{U} \mathbf{U}
$$

where $\mathbf{K}_{y}$ and $\mathbf{K}_{U}$ are diagonal weight matrices.

The minimization of $J$ for a given current state is equivalent to

$$
\min _{\mathbf{U}}[\mathbf{U}^{T} \underbrace{\left(\mathbf{E}^{T} \mathbf{K}_{Y} \mathbf{E}+\mathbf{K}_{U}\right)}_{\mathbf{H}_{c}} \mathbf{U}+2 \underbrace{(\mathbf{D} \mathbf{y}+\mathbf{F}-\mathbf{w})^{T} \mathbf{K}_{Y} \mathbf{E}}_{\mathbf{d}^{T}} \mathbf{U}]
$$


This minimization is a case of quadratic programming (QP). Constraints on cable tension limits and rates of change can be easily added

$$
\left.\begin{array}{c}
\boldsymbol{\tau}_{\text {min }} \leqslant \boldsymbol{\tau} \leqslant \boldsymbol{\tau}_{\text {max }} \\
\left|\dot{\tau}_{i}\right| \leqslant \dot{\tau}_{\text {max }}
\end{array}\right\} \Rightarrow \mathbf{A}_{\text {ineq }} \mathbf{U} \leqslant \mathbf{b}_{\text {ineq }}
$$

Accordingly, at each time step, a QP problem is solved in order to determine the optimal control output $\boldsymbol{\tau}(t)$. This problem is stated as

$$
\begin{aligned}
\min _{\mathbf{U}} & \frac{1}{2} \mathbf{U}^{T} \mathbf{H}_{c} \mathbf{U}+\mathbf{d}^{T} \mathbf{U} \\
& \text { s.t. } \quad \mathbf{A}_{\text {ineq }} \mathbf{U} \leqslant \mathbf{b}
\end{aligned}
$$

The QP problem (18) can be efficiently solved with the Interior Point method implemented in the MATLAB function quadprog.

The optimal argument $\mathbf{U}(t)=\left[\boldsymbol{\tau}^{T}(t), \boldsymbol{\tau}^{T}(t+\Delta t), \ldots, \boldsymbol{\tau}^{T}(t+(N-1) \Delta t)\right]^{T}$ contains all vectors of future control outputs over the control prediction horizon. The controller applies the first sample of the obtained sequence and maintains this action until the next time step. After that, the algorithm is repeated. The

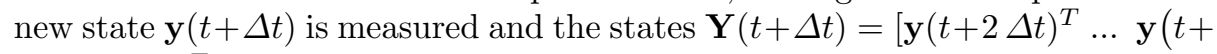
$\left.(N+1) \Delta t)^{T}\right]^{T}$ are estimated and optimized taking $\mathbf{U}(t+\Delta t)$ as argument. The procedure is repeated until $t \geqslant t_{f}$.

\section{Simulation Results}

This section presents simulation results that compare the performances obtained with the three control strategies presented in Section 3. The context of these simulations is the project Hephaestus [21] where a large-dimension CDPR is intended to automatize several tasks in the construction and maintenance of building facades. The main task of the CDPR is the installation of curtain wall modules. The robot workspace is a rectangular region in front of the building facade. Thereby, the CDPR mobile platform can get curtain wall modules on the ground and position them where needed on the building facade. Since the CDPR will operate in an outdoor environment, it will be subjected to external disturbances. One of the main concerns is the incidence of wind gusts. For this reason, the simulations presented in this section focus on external disturbance rejection performances of the proposed control strategies. An impulsive disturbance is applied and the response of the CDPR is analyzed. Note that the simulated trajectory is relatively short. However, the simulation of a longer trajectory would not affect the results which highlight the disturbance rejection capabilities of each control strategy.

The initial and final positions are depicted in Fig. 2. The path between these two positions is a straight line segment. The trajectory is the fastest possible respecting upper bounds on linear velocities, accelerations and jerks. These bounds are $0.3 \mathrm{~m} / \mathrm{s}, 0.3 \mathrm{~m} / \mathrm{s}^{2}$ and $1.0 \mathrm{~m} / \mathrm{s}^{3}$, respectively. The resulting trajectory has continuous derivatives up to the acceleration level. The desired orientation of the 
platform is constant along the trajectory. An impulsive disturbance $\mathbf{f}_{d}$ is applied at the instant $t=2 \mathrm{~s}$. This impulsive wrench is $\mathbf{f}_{d}=\left[\begin{array}{llllll}55 & 55 & 550 & 0 & 0 & 0\end{array}\right]^{T}$ $(\mathrm{N})$ and it is applied at the reference point of the platform.

The CDPR configuration (cable drawing points, cable-platform attachments, and cable arrangement) can also be seen in Fig. 2. Moreover, the parameters of the CDPR dynamic model are the following: $\tau_{\min }=100 \mathrm{~N}, \tau_{\max }=14 \mathrm{kN}$, $m_{p}=1000 \mathrm{~kg},[\mathbf{c}]_{\mathcal{F}_{p}}=\left[\begin{array}{lll}0 & 5 & 0\end{array}\right]^{T} \mathrm{~m}, \mathbf{I}=\operatorname{diag}\left(\left[\begin{array}{lll}400 & 100 & 400\end{array}\right]\right) \mathrm{kg} \cdot \mathrm{m}^{2}$.

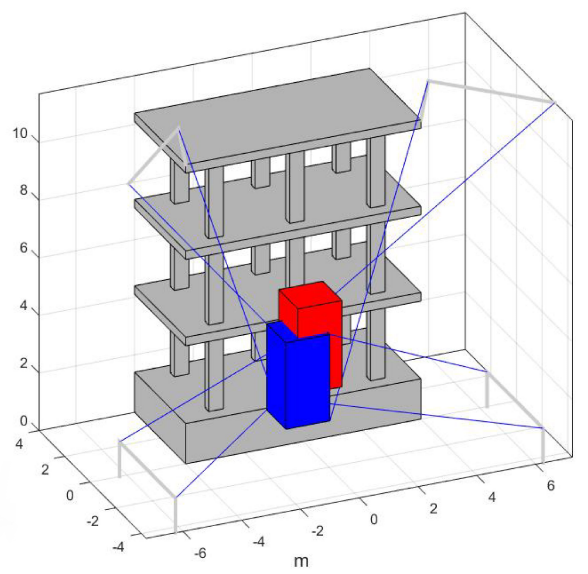

Fig. 2. Initial and final positions of the simulated trajectory

In the following, the obtained results with the three proposed motion control strategies of Section 3 are presented and discussed. Control parameters used for the simulations are the following: $\mathbf{K}_{p}=71400 \mathbf{I}_{8}, \mathbf{K}_{i}=71400 \mathbf{I}_{8}, \mathbf{K}_{d}=71400 \mathbf{I}_{8}$, $\mathbf{C}_{e}=2 \times 10^{-3} \mathbf{I}_{6}, \mathbf{C}_{d}=36 \mathbf{I}_{6}, \mathbf{Q}=40 \mathbf{I}_{6}, \mathbf{K}=0.2 \mathbf{I}_{6}, N=20, \Delta_{t}=6 \times 10^{-4}$, $\Delta_{r}=5 \times 10^{-1}, K_{Y p}=6 \times 10^{9}, K_{Y v}=1 \times 10^{-2}, \mathbf{K}_{U}=2 \times 10^{-5} \mathbf{I}_{8 N}$.

Scalar $K_{Y p}$ are the components of $\mathbf{K}_{Y}$ related to pose errors, whereas $K_{Y v}$ is related to velocity errors. Value of $\Delta_{t}$ is used in (5) as $\Delta$ for translational inputs of $\operatorname{sat}(s)$, and $\Delta_{r}$ is used for rotational inputs.

Figure 3 shows the evolution versus time of the norms of the translational and rotational errors. All the control strategies are able to cancel the tracking error caused by the applied external disturbance but PID+ presents an oscillatory behavior and increased settling time. SMC responds faster and without oscillations. MPC presents the fastest response, resulting in the smallest tracking errors along the whole trajectory.

The histograms of Fig. 4 present a performance comparison on different aspects. Let $e_{t}$ be the 2-norm of the translational error. Histogram (a) quantifies the maximum value $e_{t}$ over the trajectory. Histogram (b) compares the RMS 

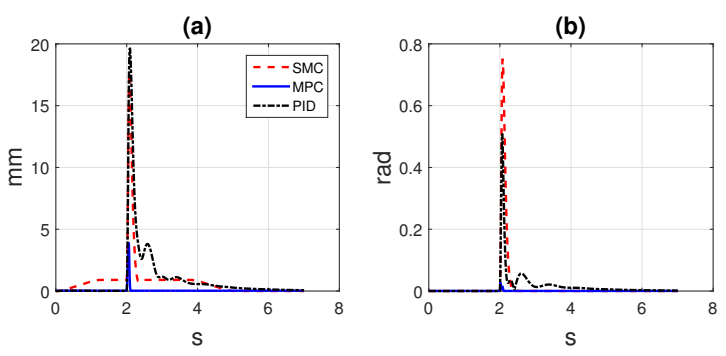

Fig. 3. Norm of the (a) translational and (b) rotational errors

value of $e_{t}$ along the trajectory. Taking these two performance measures, MPC leads to the smallest error.
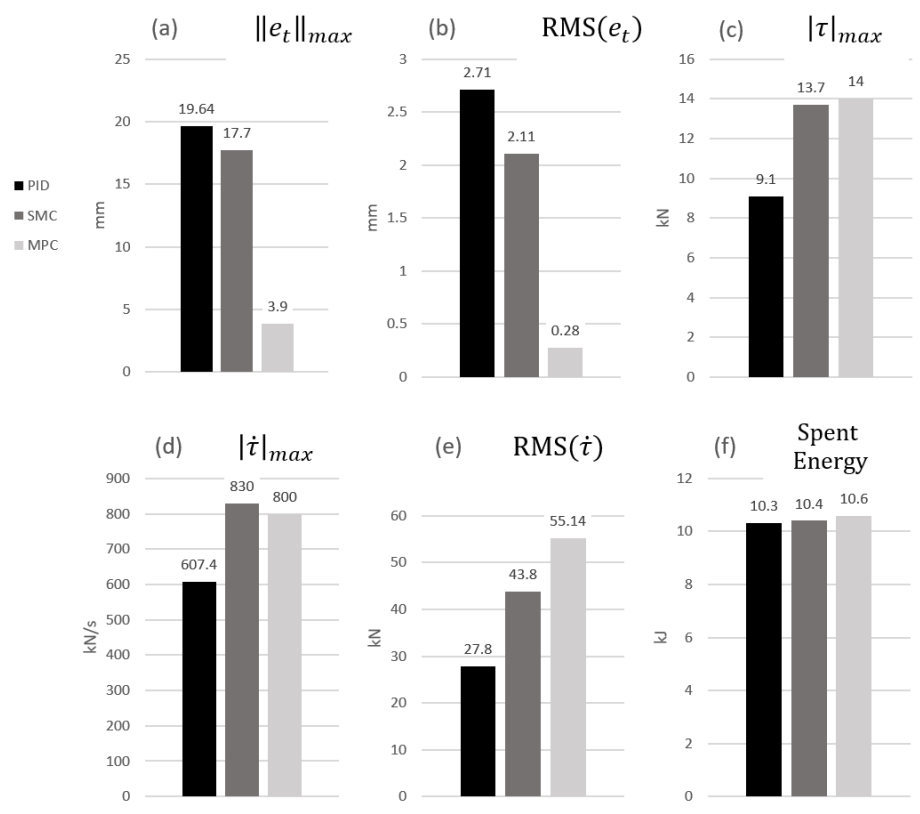

Fig. 4. Comparative results: (a) maximum error, (b) error RMS, (c) maximum cable tensions, (d) maximum cable tension derivatives, (e) RMS of cable tension derivatives, and (f) consumed energy.

Regarding cable tension values, as shown in Fig. 4-(c), MPC demands the maximum allowed value $\tau_{\max }=14 \mathrm{kN}$. This is also shown in Fig. 5, which depicts the cable tensions near the instant of application of the impulsive disturbance. Indeed, as discussed earlier, the main advantage of MPC is that the controller takes into account the constraints of the system and optimizes the control actions 
in order to reduce the tracking errors. Here, the maximum allowed cable tension is an active constraint just after the impulsive disturbance is applied. In the case of SMC, the maximum tension value is $13.7 \mathrm{kN}$ along the trajectory which indicates that this controller response is close to the largest admissible value $\tau_{\max }=14 \mathrm{kN}$. If higher gains were used, the wrench $\mathbf{f}$ would then be unfeasible. In order to not exceed $\tau_{\max }$, the strategy described in Eq. (9) would be necessary and the resulting wrench would not be equal to $\mathrm{f}$. However, the MPC strategy does not lead to this risk. The PID + controller has $\left|\tau_{\max }\right|=9.1 \mathrm{kN}$. This relatively low tension is a consequence of the use of small gains $\mathbf{K}_{p}$. Indeed, small gains were used because larger gains lead to high frequency oscillations of cable tensions. For instance, increasing the gains of less than 1\% with this strategy, $\operatorname{RMS}(\dot{\tau})$ is equal to $86 \mathrm{kN}$ (more details about this measure are discussed in the following paragraph).

The time derivative of the cable tensions is a measure of the aggressiveness of the control action. Large values of this variable may excite high frequency dynamics which are difficult to control. Figure 4-(d) presents the maximum derivative of cable tensions over the trajectory considering all cables. Mathematically, values in Fig. 4-(d) are equal to $\max _{t}\left(\max _{i}\left|\dot{\tau}_{i}(t)\right|\right)$. The smallest maximum cable tension derivative is obtained for the PID+, which is an advantage of this method. SMC is the most aggressive controller considering this performance measure. The proposed MPC strategy constraints this variable (Eq. (17)). Therefore, any value can be imposed independently of the rest of the controller parameters. In simulations, the value used is $800 \mathrm{kN} / \mathrm{s}$ and Fig. 4-(d) shows that this value is reached. Fig. 4-(e) presents the values of the maximum RMS value of $\dot{\tau}_{i}$ considering all cables $i=1, \ldots 8$. More precisely, Fig. 4 (e) presents values of $\max _{i}\left(\operatorname{RMS}\left(\dot{\tau}_{i}\right)\right)$. MPC presents the largest RMS of the cable tension derivatives. Note that the MPC optimization cannot take as constraint variables that depends on the system states beyond the prediction horizon. The prediction horizon covers only a small part of the whole trajectory over which the RMS values are calculated.

(a)

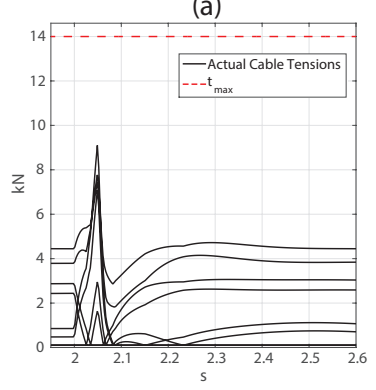

(b)

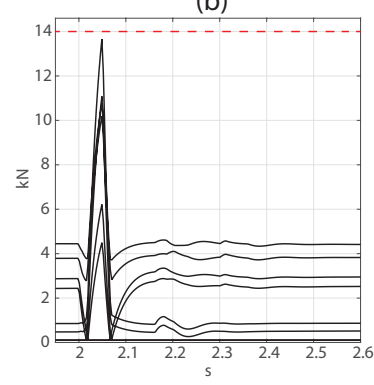

(c)

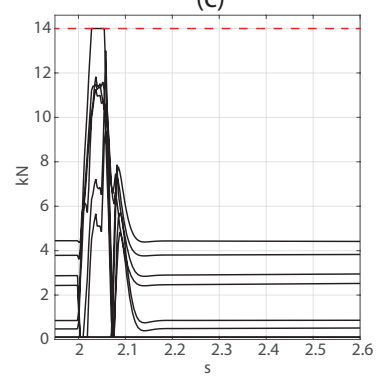

Fig. 5. Simulated cable tensions for (a) PID + Controller, (b) Sliding Mode Controller and (c) Model Predictive Control. 
The consumed energy over the trajectory (Fig.4-(f)) is roughly the same for all the control schemes. Nevertheless, MPC leads to the highest consumption. The consumed energy is calculated as $\int_{t_{i}}^{t_{f}}\left|\mathbf{i}(t)^{T} \boldsymbol{\tau}(t)\right| d t$.

\section{Conclusions and Future Work}

An MPC strategy was proposed for large-dimension CDPRs. Considering that the robot works at low velocities, a linear MPC approach was selected. A linear time invariant system is considered along the MPC optimization horizon. MPC allows to minimize the error between the desired trajectory and the predicted positions while taking into account constraints on cable tensions. Since cable tensions are the argument of this optimization, actuation redundancy resolution is integrated within the MPC optimization. Simulation results validate these advantages by comparing the disturbance rejection performances of the proposed MPC strategy with two other commonly used controllers (PID+ and SMC). MPC yields the smallest errors when compared to these two other controllers. In future works, the modeling errors due to the assumption of a linear time invariant system should be quantified. Indeed, the non-linear dynamics of the CDPR will differ from the proposed linear time invariant system according to the mobile platform dynamics and the MPC optimization horizon. These modeling errors may be predicted and the relevance of the proposed linear MPC may be evaluated in a general case. Additionally, experimental tests should be conducted to validate the simulations presented in this paper. Experimental validation may also clarify the feasibility regarding computational burden, which is a critical aspect of using MPC for real-time control of CDPRs.

\section{ACKNOWLEDGMENT}

The research leading to these results has received funding from the European Union's H2020 Programme (H2020/2014-2020) under grant agreement No. 732513 (Hephaestus project).

\section{References}

1. R. L. Williams, P. Gallina, and J. Vadia, "Planar translational cable-direct-driven robots," Journal of Robotic Systems, vol. 20, no. 3, pp. 107-120, 2003.

2. M. H. Korayem, H. Tourajizadeh, and M. Bamdad, "Dynamic load carrying capacity of flexible cable suspended robot: Robust feedback linearization control approach," Journal of Intelligent and Robotic Systems: Theory and Applications, vol. 60, no. 3-4, pp. 341-363, 2010.

3. M. A. Khosravi and H. D. Taghirad, "Robust PID control of fully-constrained cable driven parallel robots," Mechatronics, vol. 24, no. 2, pp. 87-97, 2014.

4. A. Alikhani and M. Vali, "Sliding Mode Control of a Cable-driven Robot via Double-Integrator Sliding Surface," in International Conference on Control, Robotics, and Cybernetics, 2012. 
5. G. El-Ghazaly, M. Gouttefarde, and V. Creuze, "Adaptive terminal sliding mode control of a redundantly-actuated cable-driven parallel manipulator: CoGiRo," in Cable-Driven Parallel Robots. Springer, 2015, pp. 179-200.

6. C. Schenk, C. Masone, A. Pott, and H. H. Bülthoff, "Application of a DifferentiatorBased Adaptive Super-Twisting Controller for a Redundant Cable-Driven Parallel Robot," in Cable-Driven Parallel Robots. Springer, 2018, pp. 254-267.

7. M. Zeinali and A. Khajepour, "Design and Application of Chattering-Free Sliding Mode Controller to Cable-Driven Parallel Robot Manipulator: Theory and Experiment," in Volume 2: 34th Annual Mechanisms and Robotics Conference, Parts A and B. ASME, jan 2010, pp. 319-327.

8. C. Gosselin and M. Grenier, "On the determination of the force distribution in overconstrained cable-driven parallel mechanisms," Meccanica, vol. 46, no. 1, pp. 3-15, 2011.

9. M. Gouttefarde, J. Lamaury, C. Reichert, and T. Bruckmann, "A Versatile Tension Distribution Algorithm for $n$ - DOF Parallel Robots Driven by $n+2$ Cables," IEEE Transactions on Robotics, vol. 31, no. 6, pp. 1444-1457, 2015.

10. E. F. Camacho and C. B. Alba, Model predictive control. Springer Science \& Business Media, 2013.

11. J. M. Maciejowski, Predictive control: with constraints. Pearson education, 2002.

12. L. Cuvillon, E. Laroche, J. Gangloff, and M. De Mathelin, "GPC versus $\mathrm{H} \infty$ control for fast visual servoing of a medical manipulator including flexibilities," in International Conference on Robotics and Automation - ICRA. IEEE, 2005, pp. 4044-4049.

13. G. Buondonno, F. Patota, H. Wang, A. De Luca, and K. Kosuge, "A model predictive control approach for the Partner Ballroom Dance Robot," in Robotics and Automation (ICRA), 2015 IEEE International Conference on. IEEE, may 2015, pp. $774-780$.

14. A. Chemori and M. Alamir, "Multi-step limit cycle generation for Rabbit's walking based on a nonlinear low dimensional predictive control scheme," Mechatronics, vol. 16, no. 5, pp. 259-277, 2006.

15. J. Albus, R. Bostelman, and N. Dagalakis, "The NIST robocrane," Journal of Field Robotics, vol. 10, no. 5, pp. 709-724, 1993.

16. M. Gouttefarde, J.-F. Collard, N. Riehl, and C. Baradat, "Geometry selection of a redundantly actuated cable-suspended parallel robot," IEEE Transactions on Robotics, vol. 31, no. 2, pp. 501-510, 2015.

17. G. Meunier, B. Boulet, and M. Nahon, "Control of an overactuated cable-driven parallel mechanism for a radio telescope application," IEEE transactions on control systems technology, vol. 17, no. 5, pp. 1043-1054, 2009.

18. X. Tang and R. Yao, "Dimensional Design on the Six-Cable Driven Parallel Manipulator of FAST," Journal of Mechanical Design, vol. 133, no. 11, 2011.

19. B. Paden and R. Panja, "Globally asymptotically stable pd+' controller for robot manipulators," International Journal of Control, vol. 47, no. 6, pp. 1697-1712, 1988.

20. J. Lamaury and M. Gouttefarde, "Control of a Large Redundantly Actuated CableSuspended Parallel Robot," in International Conference on Robotics and Automation. IEEE, 2013, pp. 4644-4649.

21. [Online]. Available: www.hephaestus-project.eu 